\title{
Endoscopic Repair of Frontal Sinus Cerebrospinal Fluid Leaks after Firearm Injuries: Report of Two Cases
}

\author{
Camilo Reyes $^{1} \quad$ C. Arturo Solares ${ }^{2,3}$ \\ ${ }^{1}$ Department of Otolaryngology, Universidad del Rosario, \\ Bogota, Colombia \\ 2 Department of Otolaryngology, Georgia Regents University, \\ Augusta, Georgia, United States \\ ${ }^{3}$ Center for Skull Base Surgery, Georgia Regents University, Augusta, \\ Georgia, United States
}

\begin{abstract}
Address for correspondence C. Arturo Solares, MD, Center for Skull Base Surgery, Georgia Regents University, 1120 15th Street BP 4109, Augusta, GA 30912, United States (e-mail: csolares@gru.edu).
\end{abstract}

J Neurol Surg Rep 2015;76:e8-e12.

\section{Introduction}

Missile injuries of the anterior skull base usually occur during war or warlike situations; however, in the United States, > 100 Americans die by suicide on an average day, and $\sim 50$ of these suicides involve the use of firearms. ${ }^{1}$ The anterior skull base is the most common region of the skull base injured by a cranial missile. These missile injuries can result in cerebrospinal fluid (CSF) leaks. Most CSF leaks can be broadly classified into traumatic (most frequent), nontraumatic (tumors), spontaneous, and iatrogenic. ${ }^{2}$ The clinical presentation usually is of unilateral clear rhinorrhea that can be continuous or persis- tent. The patient can complain of a saltwater taste sensation; fewer patients experience a sweet taste sensation. Also, the halo sign (clear ring surrounding a central bloody spot on gauze) can be present.

High-resolution computer tomography (CT) is a vital diagnostic and planning tool in patients with cranial and maxillofacial trauma. CT cisternography, radionuclide cisternograms, magnetic resonance imaging (MRI), $\beta-2$ transferrin, and intrathecal fluorescein are also part of the armament used in the diagnosis of CSF leaks. Some CSF leaks require surgical treatment. The goals of surgery in patients with gunshot wounds to the head are to evacuate a hematoma, received

April 6, 2014

accepted after revision

June 28, 2014

published online

March 26, 2015
DOI http://dx.doi.org/

$10.1055 / \mathrm{s}-0034-1387194$ ISSN 2193-6366.

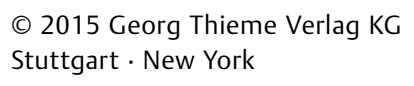

License terms

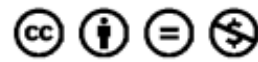



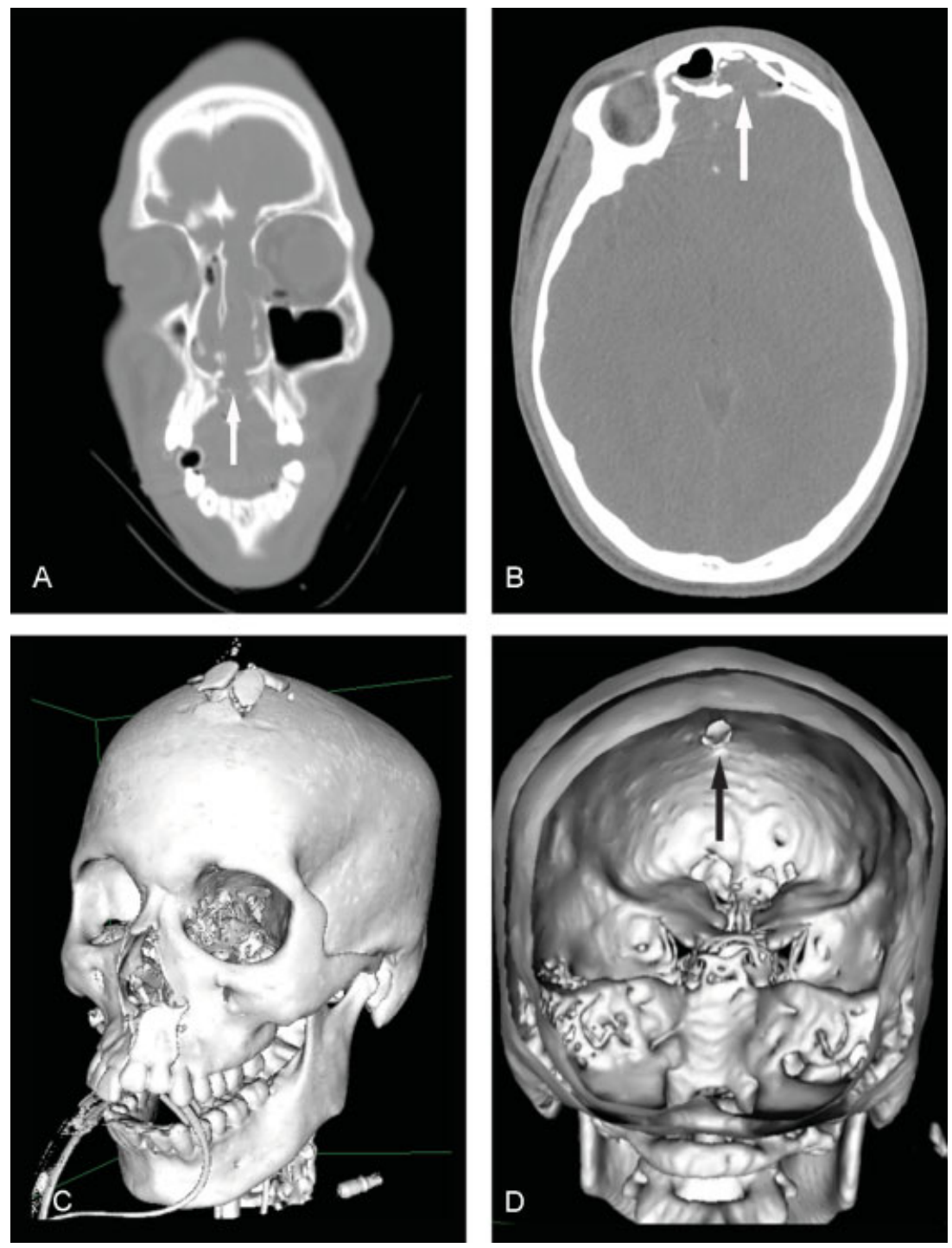

Fig. 1 (A) Noncontrast axial computed tomography consistent with a gunshot wound with entry point at the left parasagittal midline hard plate (white arrow). (B) Fracture of the inner table of both frontal sinuses with multiple fragments within the frontal sinuses and along the interhemispheric fissure (white arrow). (C) The trajectory coursed cranially through the left nasal cavity, left ethmoid air cells, left frontal lobe, and exited through the left frontal bone. (D) Posterior intracranial view shows missile trajectory entering through the skull base and exiting through the left frontal bone (arrow).

achieve hemostasis, debride the wound, provide dural closure, and repair the skull base. We present two cases of patients with CSF leak after gunshot wound to the skull base.

\section{Case Report}

\section{Case 1}

A 16-year-old young man with a history of a self-inflicted gunshot wound with point of entry through the mouth with a $3-\mathrm{cm}$ exit wound on top of the head. He had copious bleeding from the mouth and nose upon entry. Acute trauma life support protocol was undertaken. No previous significant past medical history was reported. - Fig. 1 shows the preoperative images.

The patient underwent endoscopic reduction and debridement of a frontal sinus encephalocele and CSF leak repair. An endoscopic modified Lothrop was performed to expose the lesion. The bone fragments were debrided as well as the brain tissue within the cavity. A large posterior table defect in the left frontal sinus was noted. The brain tissue was covered with Duragen (Integra Lifescience Corporation, Plainsboro, New Jersey, United States). The mucosa of the frontal sinus was removed, and abdominal fat was packed to obliterate the cavity. A mucosal graft was not feasible due to the location and extent of the defect.

To date the patient is without any complaints of clear rhinorrhea or a salty taste sensation. Nasal endoscopy shows a bilateral patent frontal sinus recess with no evidence of obstruction or drainage. Postoperative MRI shows complete frontal sinus obliteration (-Fig. 2).

\section{Case 2}

A 19-year-old female patient presented with a self-inflicted gunshot wound to the right frontal lobe with right 

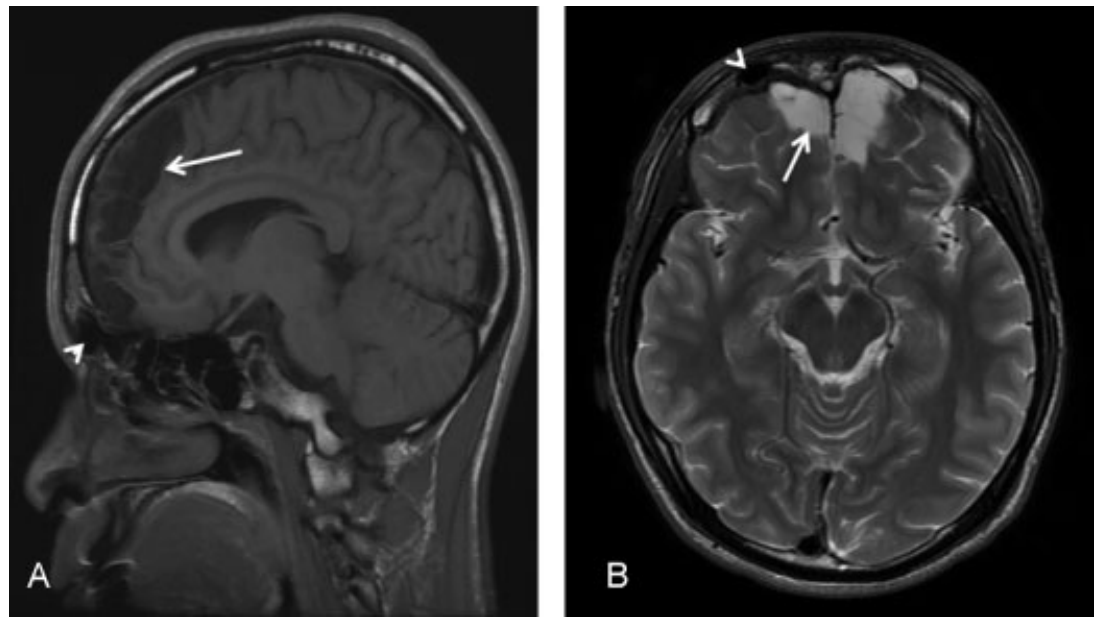

Fig. 2 (A) Sagittal magnetic resonance T1 fluid-attenuated inversion recovery scan shows postoperative result of frontal sinus obliteration and frontal sinus encephalocele repair. Bilateral frontal cortical and subcortical encephalomalacia from sequela from gunshot wound injury (arrow, $A$ and B). (B) Residual pneumatization of the left frontal sinus (arrowhead, A and B). No evidence of frontal sinus mucocele formation.

intracerebellar hematoma. Upon arrival, neurosurgeons performed a first attempt to repair the frontal sinus floor with an inner table of bone flap. They also used fibrin thrombin glue to seal the frontal skull base floor.

The bullet involved the frontal sinus, the posterior ethmoid, nasal septum, right sphenoid sinus, and medial pterygoid plate with a major bullet fragment lodged into the right, at C2-C3 vertebral level, without lesions of the cervical spine. Internal carotid artery injury was excluded ( $\mathbf{- F i g . ~} \mathbf{3}$ ).

One month after discharge, the patient was complaining of right-sided clear rhinorrhea, with no salty taste sensation in her mouth. Postfrontal craniectomy CT was done, and the patient was scheduled for endoscopic CSF leak repair ( -Fig. 4.)

The surgical procedure was done after $15 \mathrm{~mL}$ of CSF was obtained. Then $0.1 \mathrm{~mL}$ of preservative-free $10 \%$ fluorescein was mixed with $10 \mathrm{~mL}$ of CSF and slowly injected intrathecally over 30 minutes. This was followed by slow infusion of 5 $\mathrm{mL}$ of pure CSF. Uncinectomy, antrostomy, anterior and posterior ethmoidectomy, and sphenoidotomy were done. The skull base was skeletonized working in a posterior to anterior direction. Due to multiple skull base fractures, image guidance was used continuously. The sites of the CSF leak were identified at the ethmoid frontal junction anteriorly and in the posterior ethmoidal cells. Mucosa was dissected off the turbinate bone. Previous harvested abdominal fat was positioned at the site of the leaks, which resulted in cessation of the fluorescein-identified leak. Mucosal grafts were placed and positioned over the leak. Both grafts were supported with Surgicel (Ethicon, Inc., Somerville, New Jersey, United States) followed by DuraSeal (Confluent Surgical, Inc., Waltham, Massachusetts, United States). NexFoam (Hemostasis, Saint Paul, Minnesota, United States) were placed to further support the graft, and two Merocels (Medtronic, Mystic, Connecticut, United States) were placed in the right nasal cavity. To date there is no evidence of CSF leak.

\section{Discussion}

CSF is an ultrafiltrate of plasma produced by the choroid plexus that offers mechanical and immunologic protection for the brain. CSF leaks occur when the skull and its underlying dura are ruptured. A CSF rhinorrhea is defined as a fistula
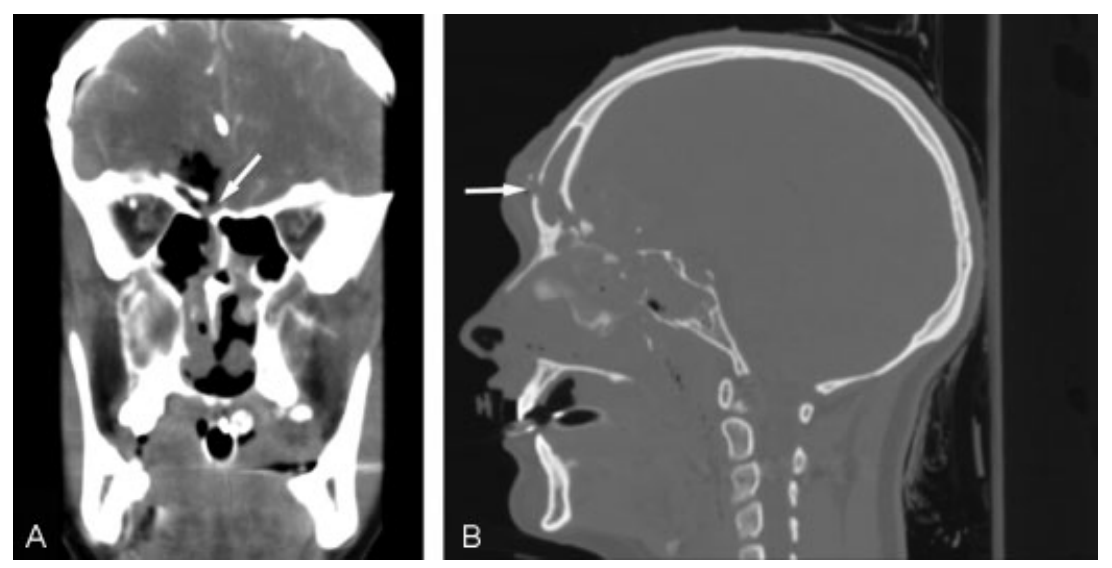

Fig. 3 (A) Coronal head computed tomography (CT) shows the skull base fracture (arrow). (B) Sagittal view of head CT shows bullet entry in the forehead through the anterior and posterior walls of the right frontal sinus (arrow). 

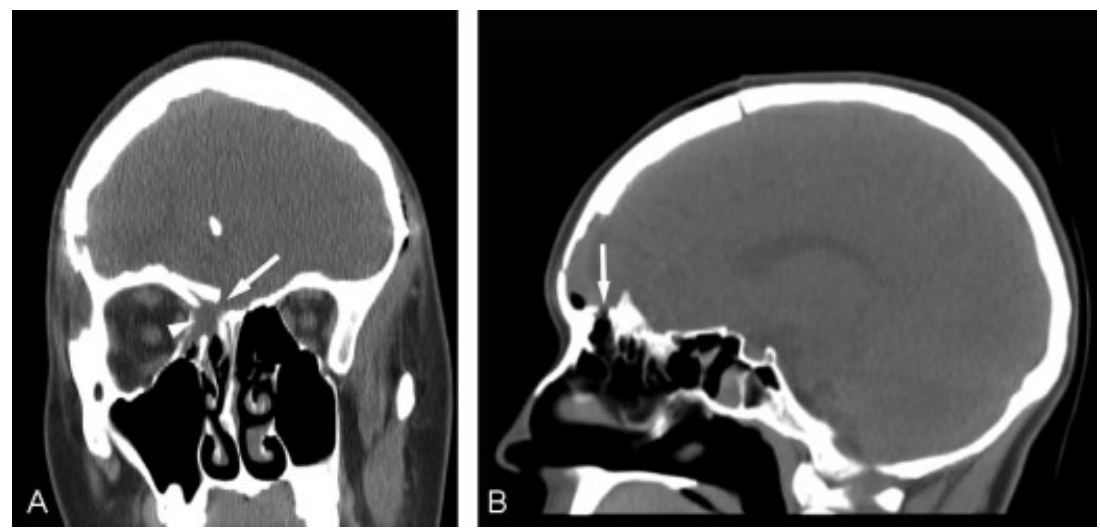

Fig. 4 (A) Coronal view of status postfrontal craniectomy. Right anterior ethmoidal air cells opacification (arrowhead) with fracture of ethmoid sinus roof (arrow). (B) Sagittal view shows fracture at the ethmoidofrontal junction (arrow).

between the dura and the skull base with CSF discharge through the nose. ${ }^{3}$ About 70 to $80 \%$ of CSF leaks are secondary to trauma and evident within the first 2 days, first week, or within the first 3 months. ${ }^{2,4}$ Energy deposited along the missile trajectory shatters the bone and lacerates soft tissues, which makes it difficult to diagnose a CSF leak upon arrival. ${ }^{5}$ Also, the surrounding edema associated with the trauma can conceal these leaks momentarily, so CSF leaks can be revealed after this edema subsides or necrosis of the surrounding tissues occurs.

The greatest concern with a CSF leak is the potential for meningitis, with a generally accepted rate of $10 \%{ }^{6}$ Approximately $35 \%$ of patients with recurrent meningitis have CSF leaks secondary to trauma. ${ }^{2}$ A missile traversing the face creates a communication between air-filled mucus-secreting cavities and the subarachnoid spaces with wound contamination through the missile entry point from oropharyngeal commensal staphylococcus, gram-negative or gram-positive rods. $^{7}$ A 2006 Cochrane Review ${ }^{8}$ concluded that no evidence indicates that prophylactic antibiotics reduce the risk of meningitis in patients with skull base fractures with active CSF leak. However, there are no data to apply this statement to patients with gunshot wounds to the head.

High-resolution CT is vital for the planning and prognosis of patients with a gunshot wound to the head; it is also the preferred method to localize the site of a skull base fracture. However, CT cisternography or MRI may be required to diagnose CSF rhinorrhea. In the presence of a skull base fracture on CT and a clinical CSF leak, there is no need for a further confirmatory test. In cases where a confirmatory test is needed, the $\beta-2$ transferrin assay is the test of choice because of its high sensitivity and specificity. ${ }^{4,9}$

Intrathecal fluorescein has been used to confirm and intraoperatively localize CSF leaks. ${ }^{2,4}$ The recommended dilution is $0.1 \mathrm{~mL}$ of $10 \%$ intravenous fluorescein in $10 \mathrm{~mL}$ of the patient's own CSF that is infused slowly over 30 minutes. A yellow fluid leaking in the nose should be visualized in the vicinity of the defect, and the use of a blue-light filter makes the sensitivity even higher.

Most CSF leaks close spontaneously within 7 to 10 days. Patients with CSF leaks $<7$ days have an $11 \%$ chance to develop meningitis versus $88 \%$ of those lasting $>7$ days. Surgical treatment is indicated when they last $>1$ or 2 weeks. $^{10}$ Patients with increased intracranial pressure, such as those who experience intracranial trauma, make hernias at points of weakness; these patients have a less successful rate of CSF leak closure and more risk of developing meningoencephalocele. ${ }^{4}$ Nonetheless, the success rate of surgical closure is $\sim 95 \%$ for CSF leaks associated with trauma. ${ }^{11}$

In patients with a gunshot wound to the head, neurosurgical intervention is usually required, so it is reasonable to repair an evident CSF leak. However, when intracranial trauma is present, the associated significant brain edema makes an early repair likely to fail because of elevated intracranial pressure.

In agreement with other authors, ${ }^{12,13}$ we believe the use of a lumbar drain does not provide an additional benefit in the treatment of CSF leaks associated with gunshot wounds to the head, especially because of the risk of overdrainage and the resultant pneumoencephalos.

Endoscopic closure of CSF leaks was first described by Wigand in 1981, and to date, it continues to be the preferred method of CSF leak closure because of its high success rate (90-97\%). ${ }^{2-4,9,10,14}$ In cases of trauma, an open approach for repair of these lesions is still favored. We present two cases where satisfactory endoscopic CSF leak repair was performed after gunshot wound to the head. The primary goal of the repair is to separate the intracranial from the extracranial spaces. In the cases presented, an endoscopic repair was favored to minimize brain manipulation of an already severely injured brain. Cranial base repair with fat and/or mucosa is utilized in other instances and highly successful. Absorbable and nonabsorbable packing is routinely used to support the repair. We favor the use of antistaphylococcal antibiotics in traumatic cases.

Postoperative recommendations are 30 degrees of head elevation, bed rest, stool softeners, antiemetics, blood pressure management, and recommendations to refrain from sneezing, nose blowing, coughing, and Valsalva maneuvers. Postoperative follow-up with CT scanning is helpful. 


\section{Conclusion}

Endoscopic closure of anterior skull base CSF leaks is now recognized as the treatment of choice for most CSF leaks. In those cases resulting from firearm injuries, the role of endoscopy is less clear. Many patients require transcranial procedures to address intracranial injuries. Treatment should be decided by the severity of neurologic deterioration throughout the emergency period and the existence or absence of associated intracranial lesions. The risk of suppurative sequelae in patients with a missile trajectory though the skull base are high, especially when the sinuses have been compromised, so the timing for surgery should be decided with great care and with a multidisciplinary approach. In selected patients in whom the primary concern is the repair of a CSF leak and there is no need to address intracranial injuries, an endoscopic endonasal repair can be safe and effective.

\section{References}

1 Miller M, Barber C, White RA, Azrael D. Firearms and suicide in the United States: is risk independent of underlying suicidal behavior? Am J Epidemiol 2013;178(6):946-955

2 Kerr JT, Chu FWK, Bayles SW. Cerebrospinal fluid rhinorrhea: diagnosis and management. Otolaryngol Clin North Am 2005; 38(4):597-611

3 Abuabara A. Cerebrospinal fluid rhinorrhoea: diagnosis and management. Med Oral Patol Oral Cir Bucal 2007;12(5):E397-E400
4 Prosser JD, Vender JR, Solares CA. Traumatic cerebrospinal fluid leaks. Otolaryngol Clin North Am 2011;44(4):857-873, vii

5 Swan KG, Swan RC. Principles of ballistics applicable to the treatment of gunshot wounds. Surg Clin North Am 1991;71(2): 221-239

6 Brodie HA. Prophylactic antibiotics for posttraumatic cerebrospinal fluid fistulae. A meta-analysis. Arch Otolaryngol Head Neck Surg 1997;123(7):749-752

7 Aarabi B, Taghipour M, Alibaii E, Kamgarpour A. Central nervous system infections after military missile head wounds. Neurosurgery 1998;42(3):500-507; discussion 507-509

8 Ratilal BO, Costa J, Sampaio C, Pappamikail L. Antibiotic prophylaxis for preventing meningitis in patients with basilar skull fractures. Cochrane Database Syst Rev 2011;(8):CD004884

9 Schoentgen C, Henaux PL, Godey B, Jegoux F. Management of posttraumatic cerebrospinal fluid (CSF) leak of anterior skull base: 10 years experience. Acta Otolaryngol 2013;133(9):944-950

10 Rice DH. Cerebrospinal fluid rhinorrhea: diagnosis and treatment. Curr Opin Otolaryngol Head Neck Surg 2003;11(1):19-22

11 Snyderman CH, Kassam AB, Carrau R, Mintz A. Endoscopic reconstruction of cranial base defects following endonasal skull base surgery. Skull Base 2007;17(1):73-78

12 Casiano RR, Jassir D. Endoscopic cerebrospinal fluid rhinorrhea repair: is a lumbar drain necessary? Otolaryngol Head Neck Surg 1999;121(6):745-750

13 Hegazy HM, Carrau RL, Snyderman CH, Kassam A, Zweig J. Transnasal endoscopic repair of cerebrospinal fluid rhinorrhea: a metaanalysis. Laryngoscope 2000;110(7):1166-1172

14 Woodworth BA, Schlosser RJ, Palmer JN. Endoscopic repair of frontal sinus cerebrospinal fluid leaks. J Laryngol Otol 2005; 119(9):709-713 